\title{
Concatenated Irregular Variable Length Coding and Irregular Unity Rate Coding
}

\author{
R. G. Maunder and L. Hanzo \\ Communications Research Group \\ School of Electronics and Computer Science, \\ University of Southampton, SO17 1BJ, UK. \\ http://www-mobile.ecs.soton.ac.uk
}




\section{Outline}

$\square$ Serial concatenations of regular and irregular codes

$\square$ EXIT chart matching

$\square$ BER and complexity performance results

$\square$ Summary 


\section{Concatenation of regular codes}
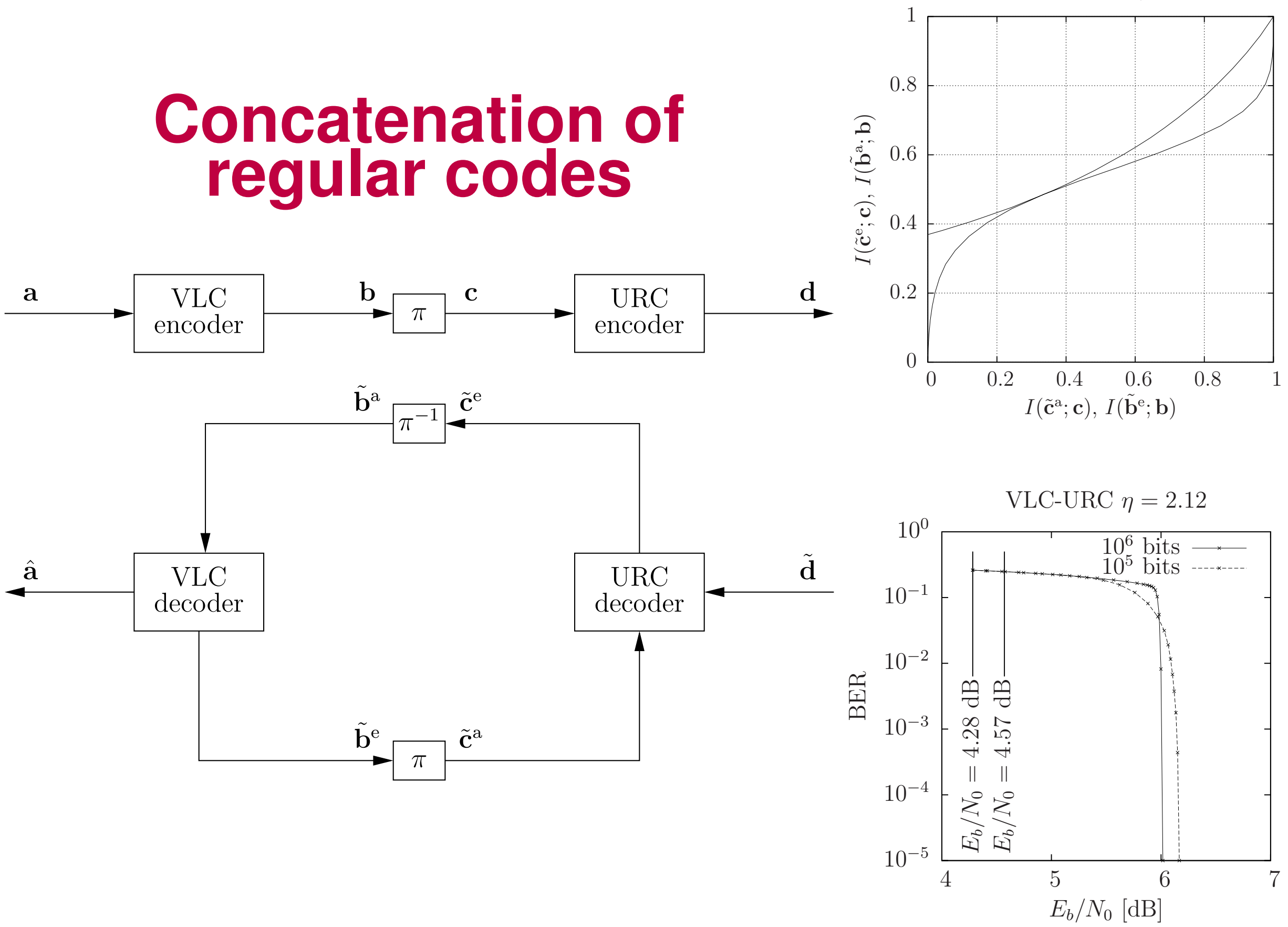


\section{Concatenation of irregular and regular codes}
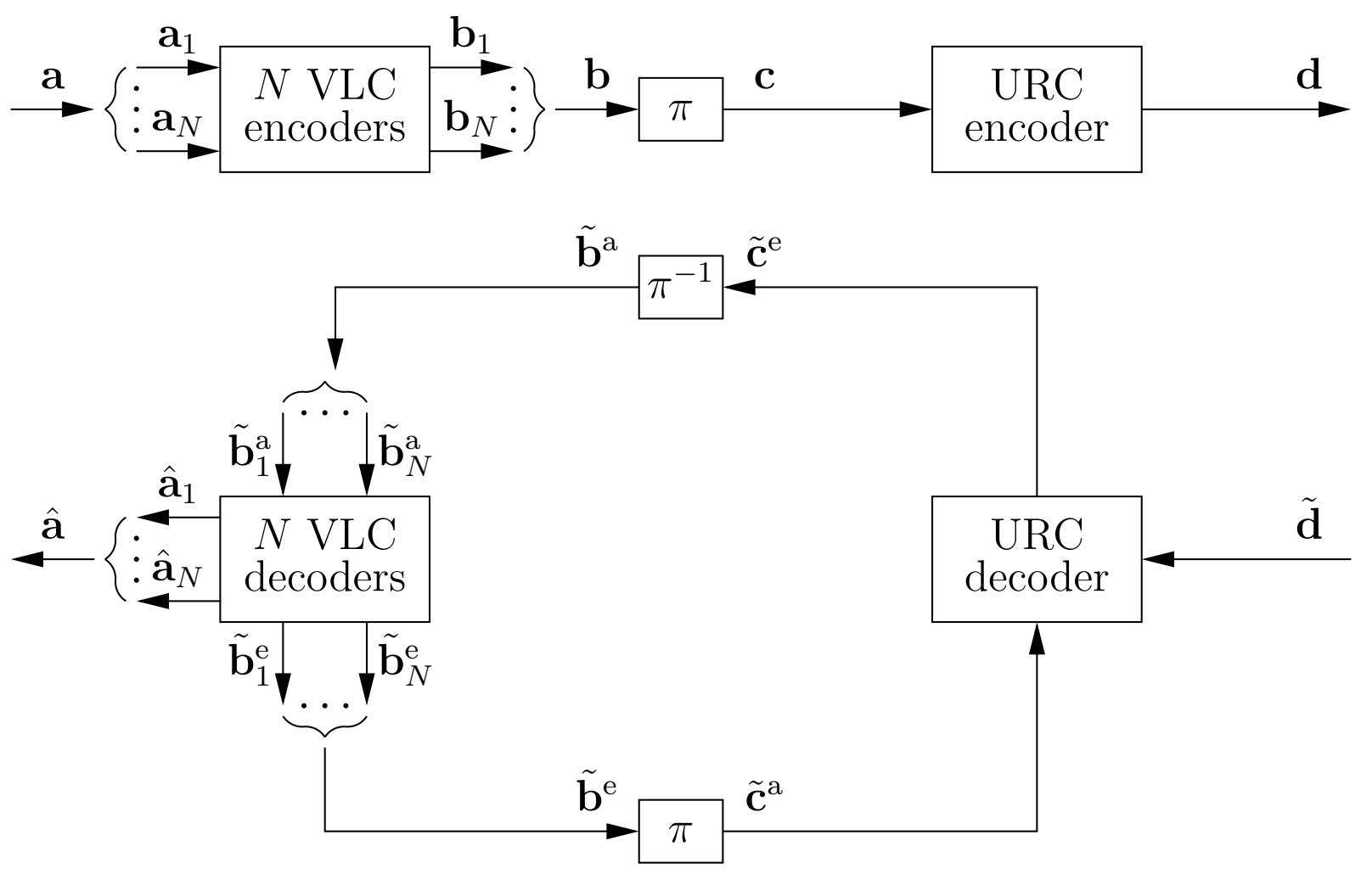
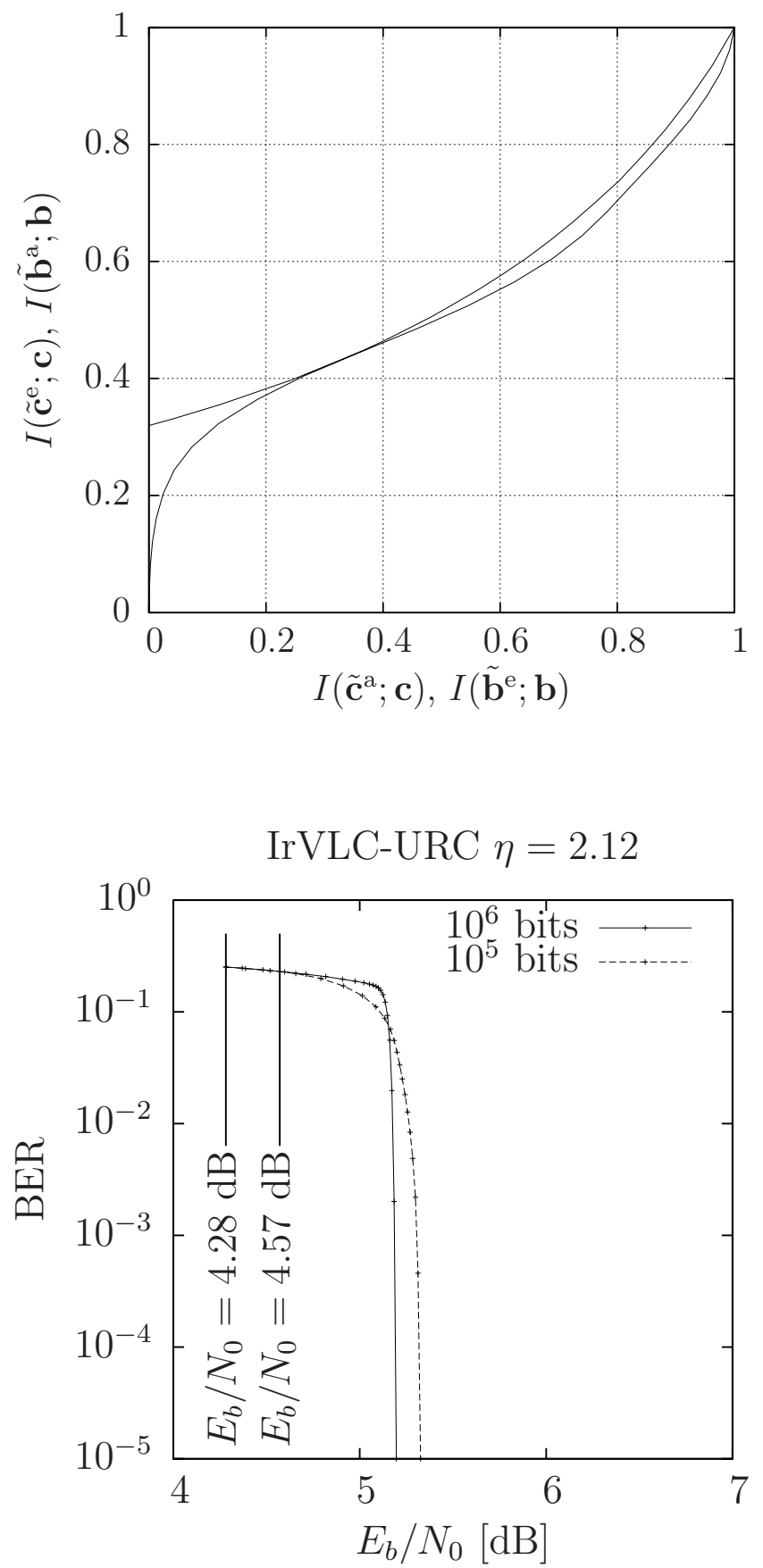


\section{Concatenation of irregular codes}
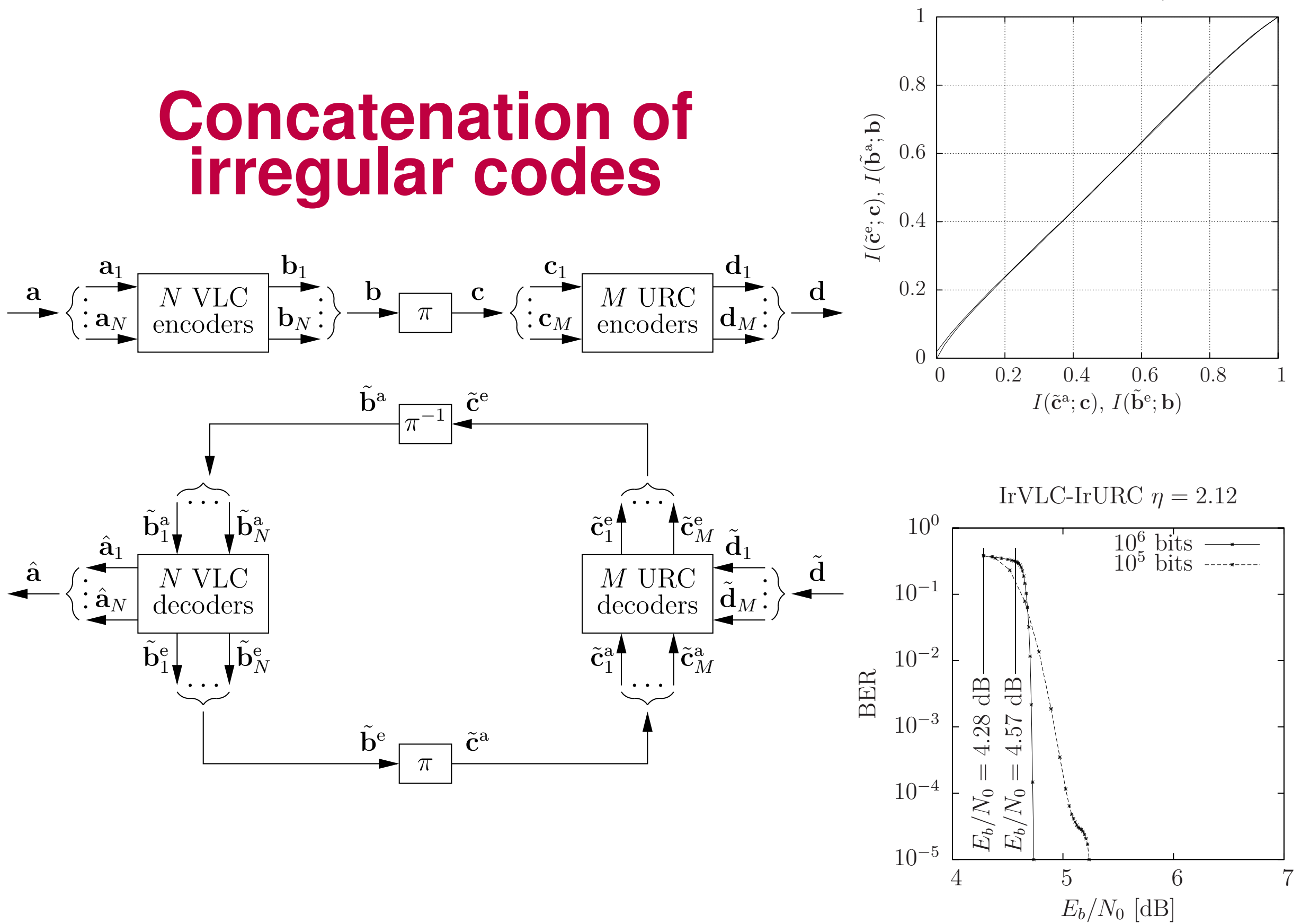

7 


\section{GA for designing component VLC codebooks}

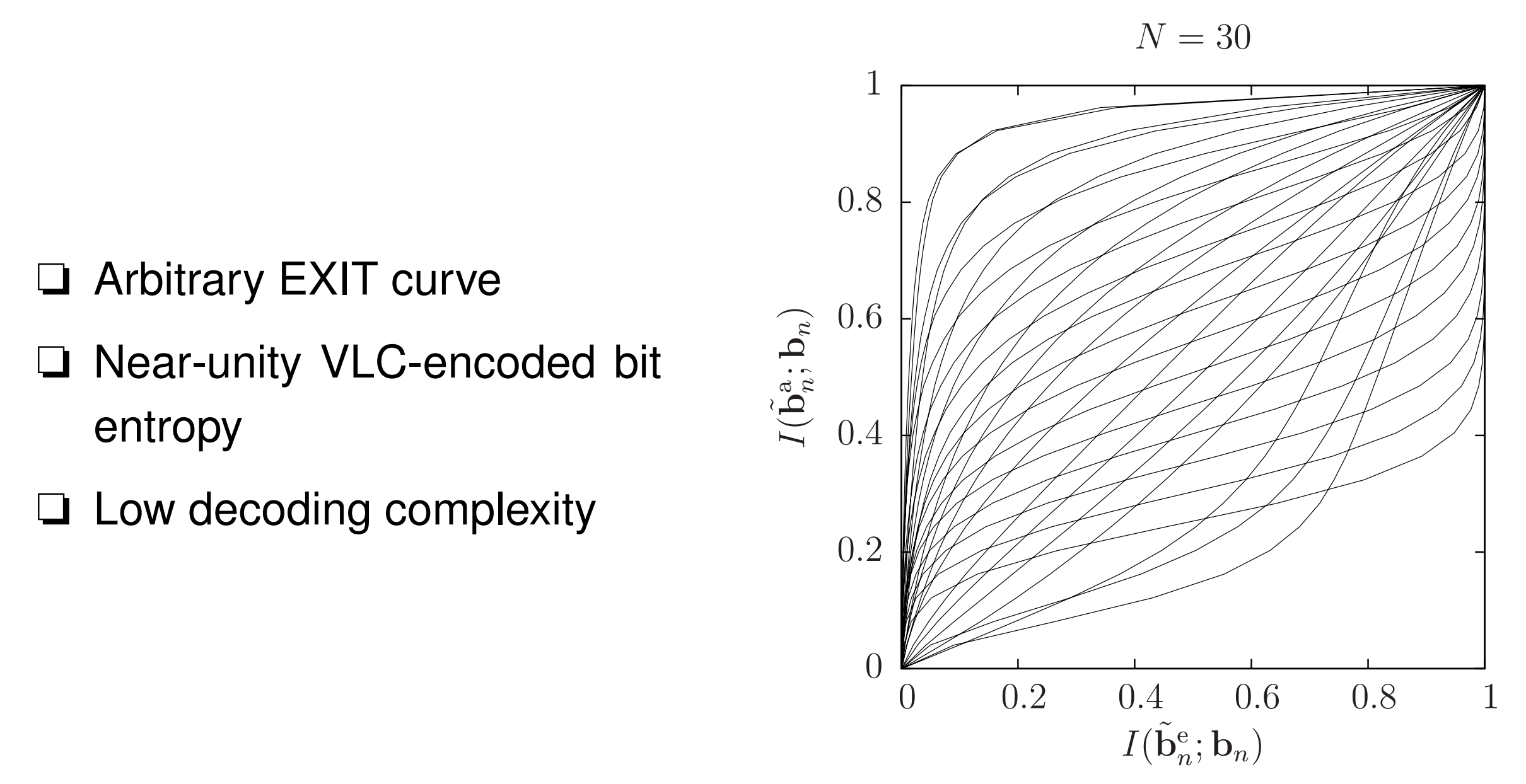




\section{Full search for selecting component URC codes}

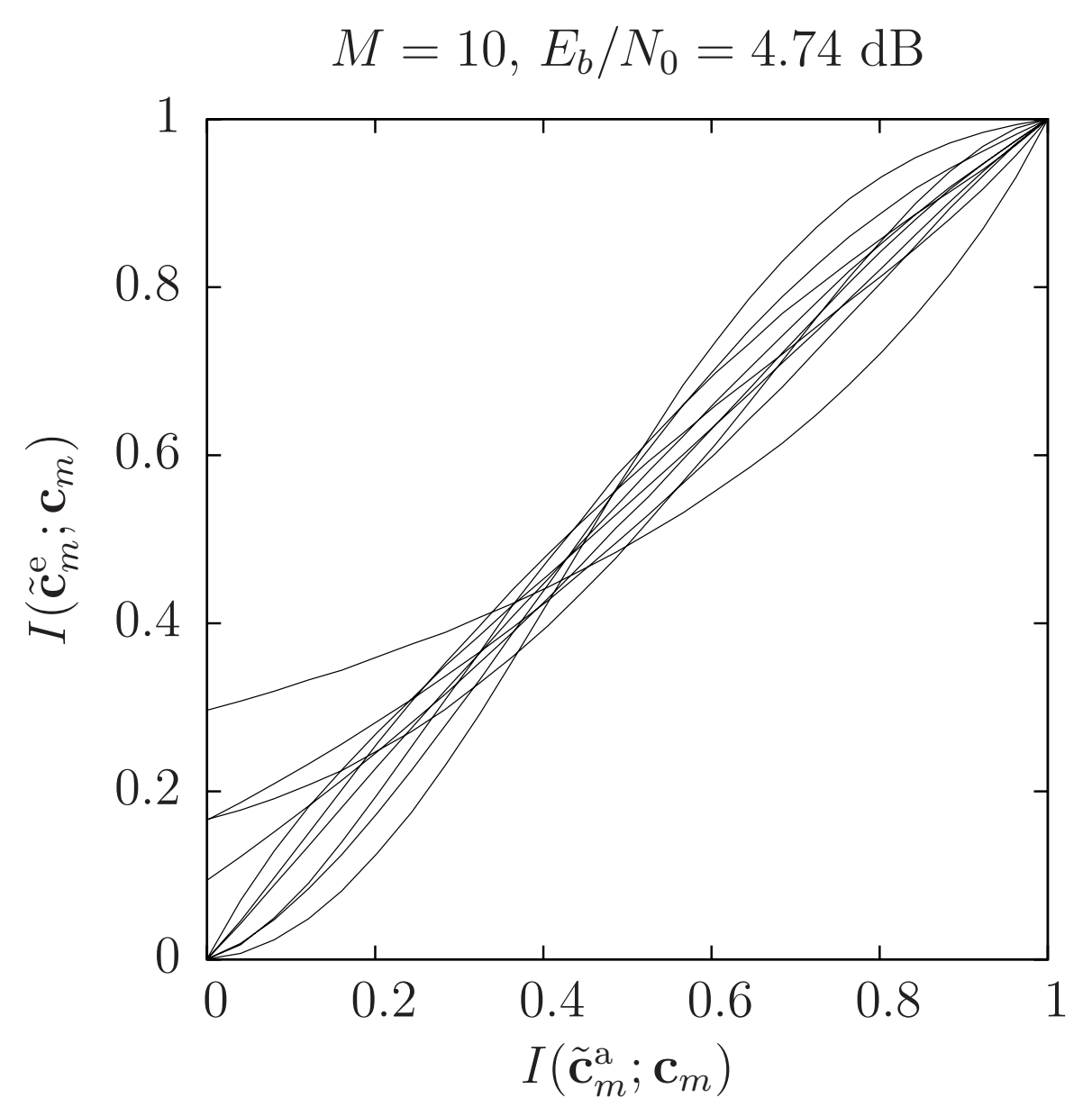

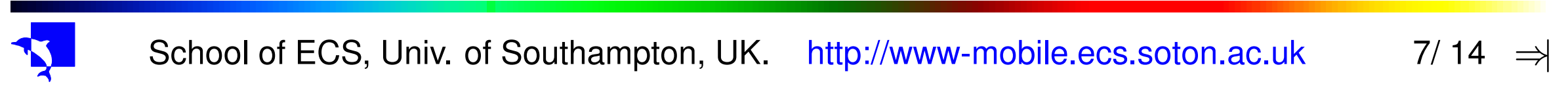




\section{EXIT chart matching}

$$
I\left(\tilde{\mathbf{x}}^{\mathrm{e}} ; \mathbf{x}\right)=f_{\mathbf{x}}\left[I\left(\tilde{\mathbf{x}}^{\mathrm{a}} ; \mathbf{x}\right)\right]
$$

IrVLC EXIT function...

$$
\begin{gathered}
f_{\mathbf{b}}=\sum_{n=1}^{N} \alpha_{n} f_{\mathbf{b}_{n}}, \\
\text { where } \\
\sum_{n=1}^{N} \alpha_{n}=1, \\
\alpha_{n} \geq 0 \forall n \in[1 \ldots N], \\
R=\sum_{n=1}^{N} \alpha_{n} R_{n} .
\end{gathered}
$$

IrURC EXIT function...

$$
f_{\mathbf{c}}=\sum_{m=1}^{M} \alpha_{m} f_{\mathbf{c}_{m}},
$$

$$
\begin{gathered}
\text { where } \\
\sum_{m=1}^{M} \alpha_{m}=1, \\
\alpha_{m} \geq 0 \forall m \in[1 \ldots M] .
\end{gathered}
$$




\section{Joint EXIT chart matching algorithm}

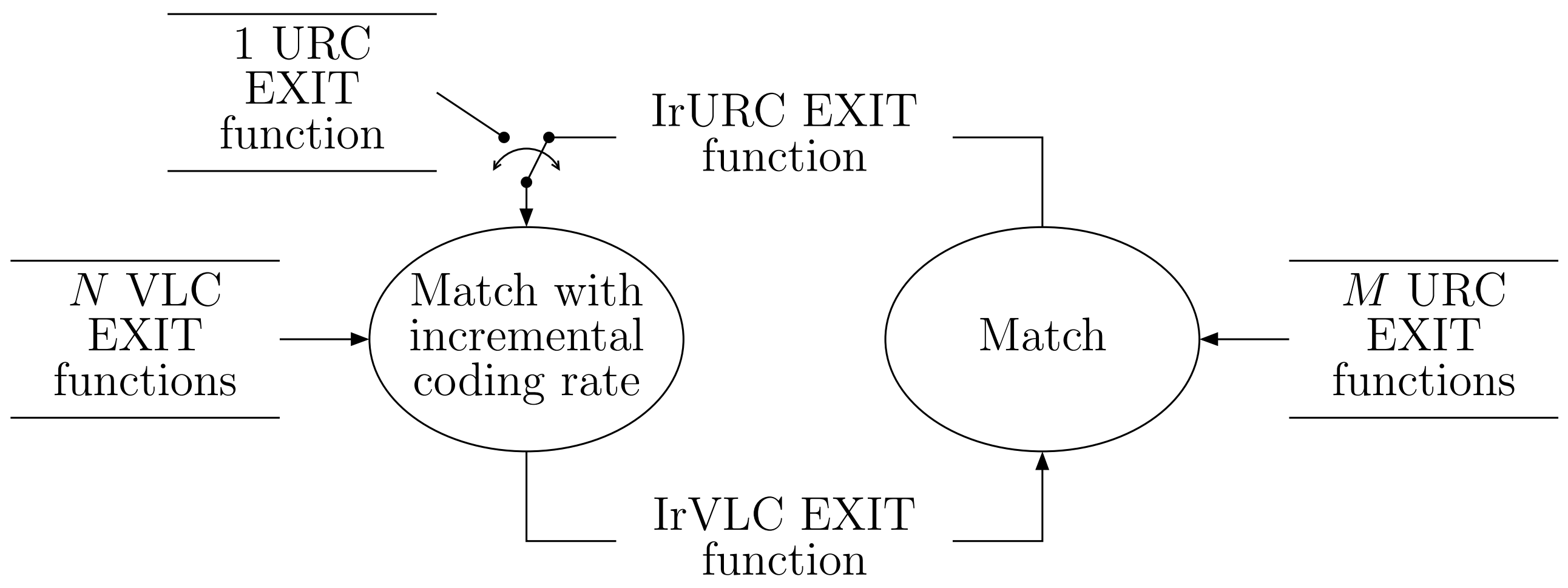




\section{EXIT chart matching accuracy}

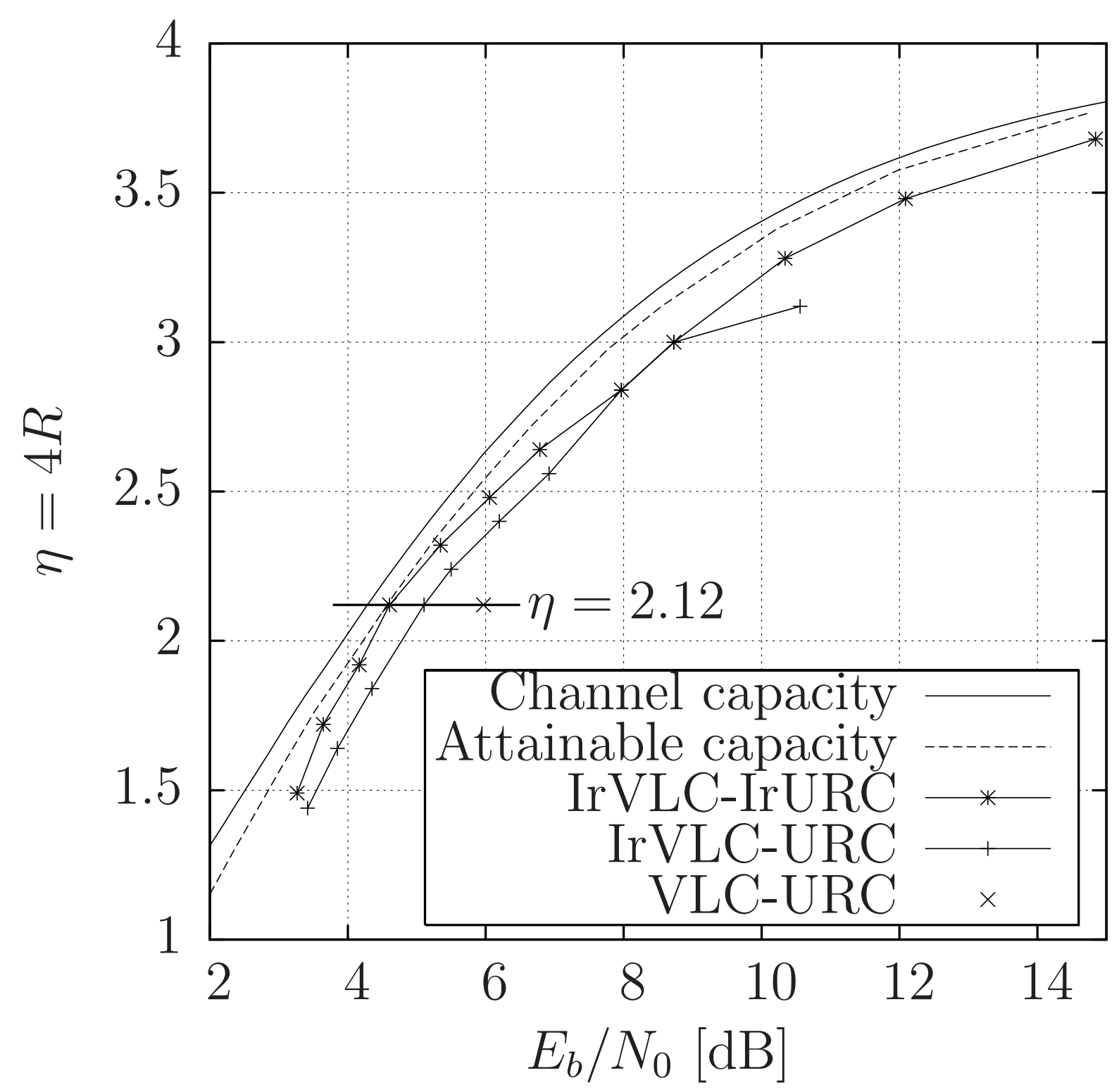




\section{EXIT charts}
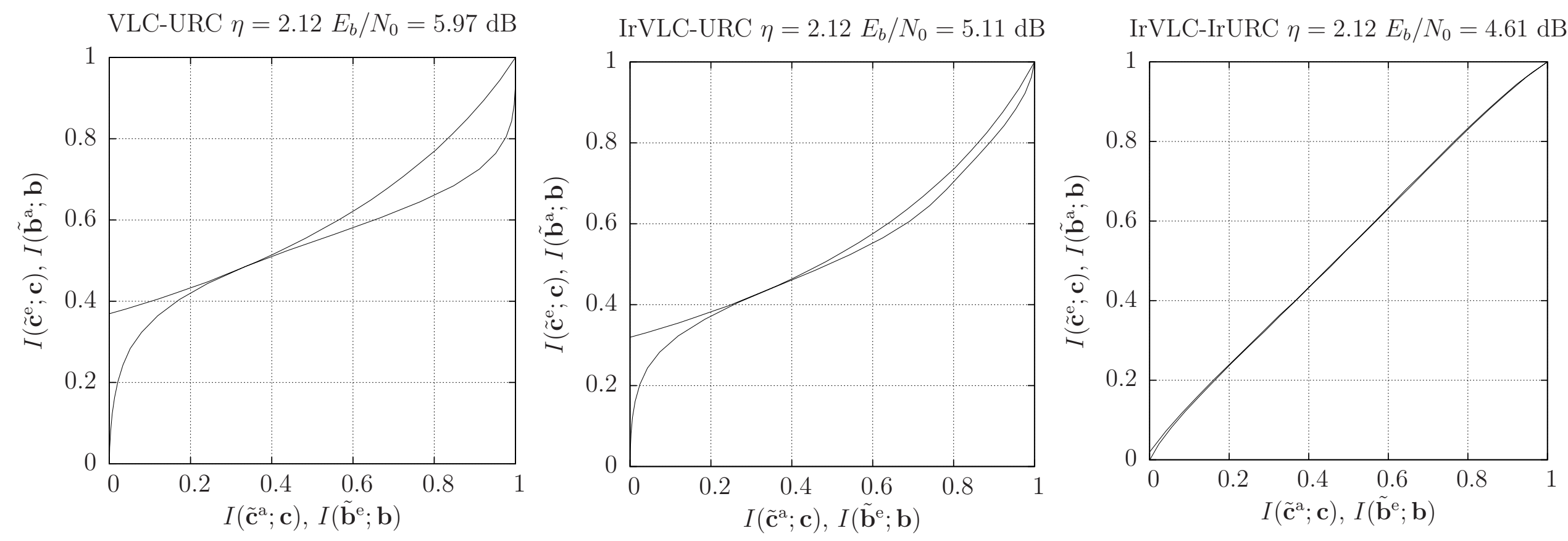

\begin{tabular}{ll|l|l|}
\hline School of ECS, Univ. of Southampton, UK. & http://www-mobile.ecs.soton.ac.uk $\quad 11 / 14 \Rightarrow 1$
\end{tabular} 


\section{BER performance}

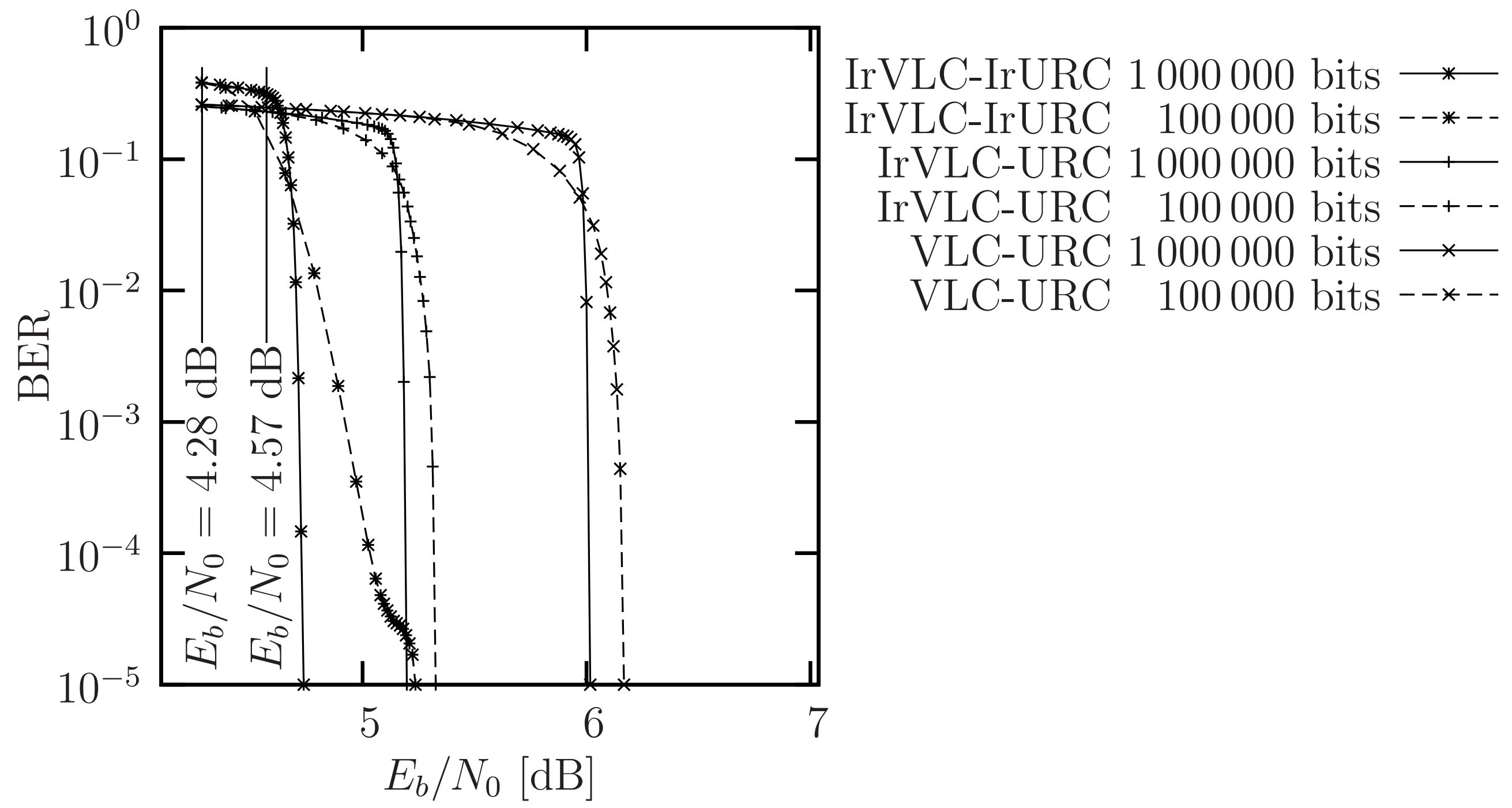

\begin{tabular}{ll|ll|l|l|} 
& School of ECS, Univ. of Southampton, UK. & http://www-mobile.ecs.soton.ac.uk & $12 / 14 \Rightarrow 1$
\end{tabular} 


\section{Decoding complexity}

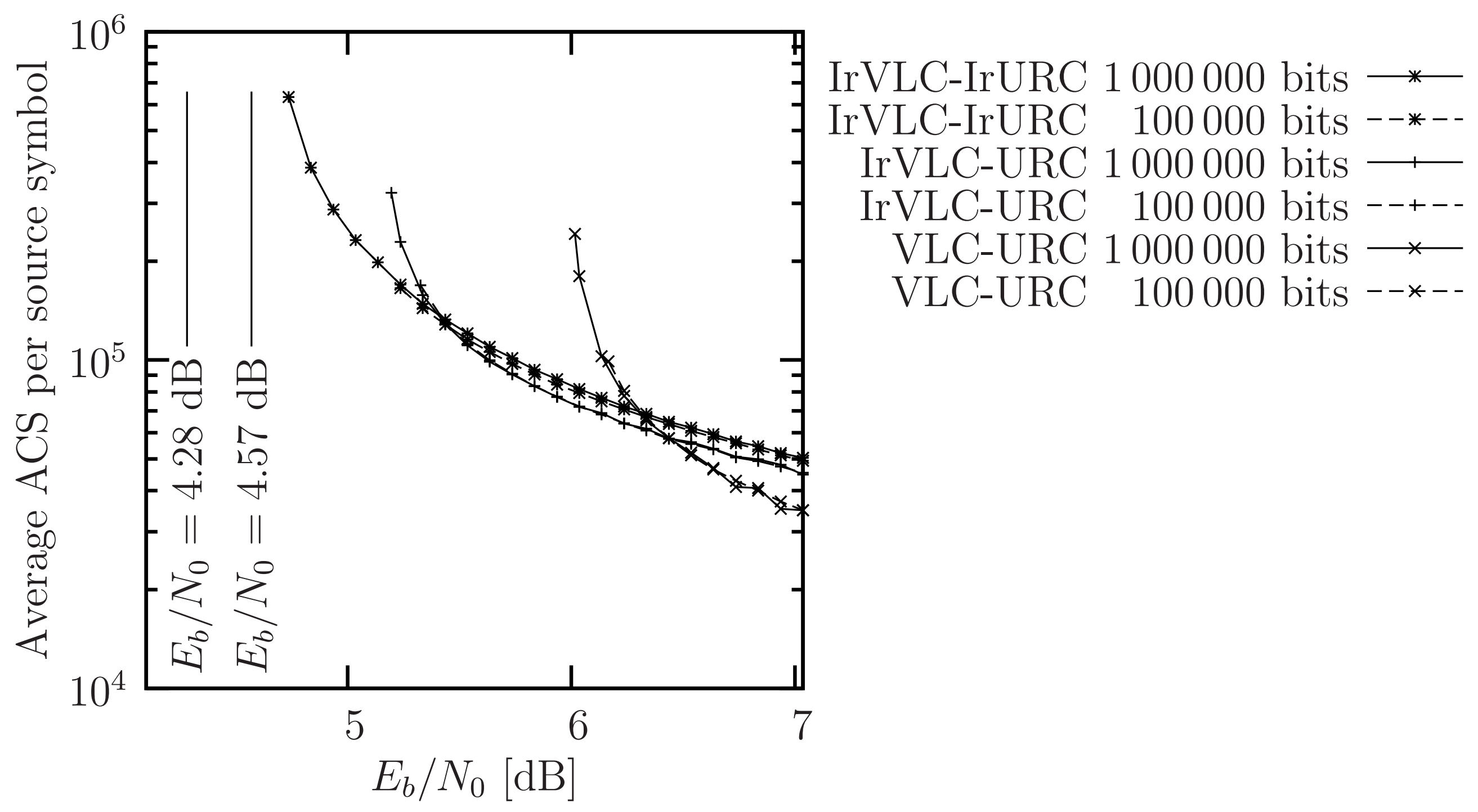

1 School of ECS, Univ. of Southampton, UK. http://www-mobile.ecs.soton.ac.uk $\quad 13 / 14 \Rightarrow$ 


\section{Summary}

$\square$ Serial concatenations of regular and irregular codes

$\square$ EXIT chart matching

$\square$ BER and complexity performance results 